\title{
ISOMORPHISM PROBLEMS FOR THE BAIRE CLASSES
}

\begin{abstract}
F. K. DAshiell, JR.
For each ordinal $\alpha>0$, the $\alpha$ th Baire class of bounded measurable functions on the topological space $S$, denoted $\mathscr{B}_{\alpha}(S)$, has an algebraic and isometric representation as a space $C\left(\Omega_{\alpha}\right)$ of all continuous functions on a totally disconnected compact space $\Omega_{\alpha}$. This representation is used to study the Baire classes from the point of view of nonseparable Banach spaces of continuous functions. It is shown that if the compact space $S$ contains an uncountable compact metrizable subset, then, for each countable ordinal $\alpha, \mathscr{B}_{\alpha}(S)$ is not isomorphic (i.e., linearly homeomorphic) to any complemented subspace of a Banach space $C(\Omega)$ for $\sigma$-Stonian $\Omega$. Since the space $\mathscr{B}_{\omega_{1}}(S)$ of all bounded Baire functions is a $C(\Omega)$ space for a certain $\sigma$-Stonian $\Omega, \mathscr{B}_{\alpha}(S)$ (for $\alpha<\omega_{1}$ ) is therefore not isomorphic to any complemented subspace of $\mathscr{B}_{\omega_{1}}(S)$.
\end{abstract}

The isomorphic invariant used here is a linear topological property of Banach spaces (see Definition 2.1) which, for spaces $C(\Omega)$ with $\Omega$ compact, is equivalent to the existence of a complementary subspace for $C(\Omega)$ in $\mathscr{B}_{1}(\Omega)$, i.e., a closed subspace $Y \subset \mathscr{B}_{1}(\Omega)$ such that $Y \cap C(\Omega)=\{0\}$ and $Y+C(\Omega)=\mathscr{B}_{1}(\Omega)$. This property passes from a space to its complemented subspaces. Thus the method consists of representing each Baire class $\mathscr{B}_{\alpha}(S)$ as $C\left(\Omega_{\alpha}\right)$, forming the first Baire class $\mathscr{B}_{1}\left(\Omega_{\alpha}\right)$ over $\Omega_{\alpha}$, and then showing that for $\alpha<\omega_{1}, C\left(\Omega_{\alpha}\right)$ is not complemented in $\mathscr{B}_{1}\left(\Omega_{\alpha}\right)$. (In fact, a slightly stronger result is proved in Corollary 2.10.) We obtain as a consequence the result of W. G. Bade [2] that $\mathscr{B}_{\alpha}([0,1])$ is not complemented as a closed subspace of $\mathscr{B}_{\alpha+1}([0,1])$ (Corollary 3.9$)$. On the other hand, it is proved in Theorem 2.11 that a compact space $\Omega$ is $\sigma$-Stonian (basically disconnected) if and only if $C(\Omega)$ is the range of a norm 1 projection from $\mathscr{B}_{1}(\Omega)$.

For any set $S$ and field $\Sigma$ of subsets of $S, B(S, \Sigma)$ denotes the sup norm closed subspace of $l^{\infty}(S)$ generated by the characteristic functions of sets in $\Sigma$. If $S$ is compact, then $\mathscr{B}_{1}(S)=B\left(S, \Sigma_{1}\right)$ for a certain field $\Sigma_{1}$ (see Theorem 1.1), and the existence of a complement for $C(S)$ in $\mathscr{B}_{1}(S)$ is equivalent to the existence of a projection of $B\left(S, \Sigma_{1}\right)$ onto $C(S)$. A topological condition on $S$ is obtained which prevents the existence of such a projection (see Definition 2.9). This condition is a consequence of an extended version of Amir's theorem [1] which provides, for certain compact spaces $S$ and fields $\Sigma$ with $C(S) \subset B(S, \Sigma)$, a lower bound for the norm of any projection from 
$B(S, \Sigma)$ onto $C(S)$ (see Theorem 2.6). This version of Amir's theorem is obtained as an application of Ditor's theorem [4] on lower bounds for norms of "averaging operators" for a continuous surjection between compact spaces.

In $\S 3$, attention is focused on the Baire classes over $[0,1]$, the results being extendable to $\mathscr{B}_{\alpha}(S)$ for more general $S$ by the BorsukDugundji theorem. We abbreviate $\mathscr{B}_{\alpha}([0,1])$ simply by $\mathscr{B}_{\alpha}$. In order to apply the above-mentioned Amir theorem to $\mathscr{B}_{\alpha}=C\left(\Omega_{\alpha}\right)$, it becomes necessary to investigate the topological structure of the maximal ideal space $\Omega_{\alpha}$. This topological structure is related to the Boolean structure of certain subfamilies of Borel sets in $[0,1]$. The relevant information is provided by results in descriptive set theory regarding the status of the Lusin separation principles at each level of the Borel heirarchy in $[0,1]$ (see Lemma 3.1).

Corollary 3.7 implies that, for countable ordinals $\alpha, \mathscr{B}_{\alpha}$ is not isomorphic to $\mathscr{B}_{\omega_{1}}$. (It is already not easy to see that $\mathscr{B}_{\alpha} \neq \mathscr{B}_{\omega_{1}}$; this is Lebesgue's classical result [13, Ch. 15].) It follows from [3, Corollary 8, p. 336] that for $\alpha>1$, there is no continuous linear injection of $\mathscr{B}_{\alpha}$ into $\mathscr{P}_{1}$. Thus there are at least three isomorphic types of Banach space represented among the Baire classes $\mathscr{B}_{\alpha}, 1 \leqq \alpha \leqq \omega_{1}$. At present it is unknown whether, for countable ordinals $\alpha>\beta \geqq 2, \mathscr{B}_{\alpha}$ and $\mathscr{B}_{\beta}$ can be isomorphic (although it is shown in Theorem 3.11 that $\mathscr{B}_{\alpha}$ and $\mathscr{B}_{\beta}$ can not be isometric Banach spaces).

The subject matter and methods of this paper grew out of the paper [2] of W. G. Bade. I would like to express my gratitude to Professor Bade for his assistance during the course of this investigation.

1. Baire classes, Baire sets, and $B(S, \Sigma)$. In this section $S$ is an arbitrary topological space.

The bounded real-valued Baire functions on $S$ are classified as follows: The functions of class 0 are the bounded continuous functions, and if the functions of class $\alpha$ are defined (which class is denoted by $\mathscr{B}_{\alpha}(S)$ ), then $\mathscr{P}_{\alpha+1}(S)$ consists of all bounded functions which are pointwise limits of convergent sequences in $\mathscr{B}_{\alpha}(S)$. For a limit ordinal $\lambda, \mathscr{B}_{A}(S)$ is defined (following Hausdorff [9, pp. 292293]) to consist of all functions which are pointwise limits both of increasing sequences and of decreasing sequences in $\mathrm{U}_{\alpha<\lambda} \mathscr{B}_{\alpha}(S)$. (This definition of $\mathscr{B}_{\lambda}(S)$ for limit ordinals $\lambda$ produces exact agreement between the classes of functions and classes of sets as given below in Theorem 1.1.) $\mathscr{P}_{\lambda+1}(S)$ then coincides with the family of all bounded pointwise limits of convergent sequences in $\bigcup_{\alpha<\lambda} \mathscr{B}_{\alpha}(S)$. For $\alpha \geqq \omega_{1}$, these classes coincide with the class of all bounded Baire functions on $S$, i.e., the smallest class of functions containing the bounded 
continuous functions and closed under pointwise convergence of bounded sequences, and clearly, $\mathscr{B}_{\omega_{1}}(S)=\bigcup_{\alpha<\omega_{1}} \mathscr{B}_{\alpha}(S)$. Thus only ordinals $\alpha \leqq \omega_{1}$ are of interest.

The Baire sets in $S$ are classified as follows: The sets of multiplicative class 0 , denoted $Z_{0}(S)$, are the "zero sets" of $S$, i.e., the sets $f^{-1}(0)$ for continuous $f: S \rightarrow \boldsymbol{R}$; the sets of additive class 0 , denoted $C Z_{0}(S)$, are the complements of zero sets. Inductively, the sets of multiplicative class $\alpha$, denoted $Z_{\alpha}(S)$, are the countable intersections from $\bigcup_{\beta<\alpha} C Z_{\beta}(S)$, and the sets of additive class $\alpha$, denoted $C Z_{\alpha}(S)$, are their complements. The sets in $A_{\alpha}(S)=Z_{\alpha}(S) \cap C Z_{\alpha}(S)$ are called the sets of ambiguous class $\alpha$. For $\alpha \geqq \omega_{1}$, these classes coincide with the $\sigma$-field of Baire sets in $S$, i.e., the smallest $\sigma$-field for which each continuous function $f: S \rightarrow \boldsymbol{R}$ is measurable. Again, only ordinals $\alpha \leqq \omega_{1}$ are of interest. (This definition of the Baire sets, although different from another common definition, is justified in the present context by Theorem 1.1.) A simple induction shows that $Z_{\alpha}(S) \cup$ $C Z_{\alpha}(S) \subset A_{\alpha+1}(S)$ and that $A_{\alpha}(S)$ is a field of sets. Also, $Z_{\omega_{1}}(S)=$ $C Z_{\omega_{1}}(S)=\bigcup_{\alpha<\omega_{1}} A_{\alpha}(S)$. In a metric space $S, Z_{0}(S)$ is just the family of closed sets, $Z_{1}(S)$ is the family of $G_{\delta}$ sets, $A_{1}(S)$ is the family of sets both $F_{\sigma}$ and $G_{\delta}$, etc.

The fundamental connection between the above classifications of Baire functions and Baire sets is given in the following theorem, which is due to Lebesque, Hausdorff, and others. This theorem was essentially known in the 20's, although there does not seem to be any one place in the literature which summarizes the relationship between sets and functions in this way. The following formulation is particularly useful in the study of Baire classes as Banach spaces of continuous functions (see Theorem 1.4).

We recall that if $\Sigma$ is a field of subsets of $S$ (not necessarily a $\sigma$-field), then $B(S, \Sigma)$ denotes the closed subspace of $l^{\infty}(S)$ generated by $\left\{k_{E}: E \in \Sigma\right.$, where $k_{E}$ is the characteristic function of the set $E$. The scalar field is assumed real.

THEOREM 1.1. For $\alpha \geqq 1, \mathscr{B}_{\alpha}(S)=B\left(S, A_{\alpha}(S)\right)$, i.e., the $\alpha$ th Baire class is the closed linear span of characteristic functions of ambiguous class $\alpha$ sets.

Proof. $\mathscr{B}_{\alpha}(S)$ consists of all bounded functions $f: S \rightarrow \boldsymbol{R}$ such that for each $t \in R,\{x \in S: f(x) \geqq t\} \in Z_{\alpha}(S)$ and $\{x \in S: f(x)>t\} \in C Z_{\alpha}(S)$ (see Hausdorff $[9, \S 43.1]$ ). Thus for each $E \in A_{\alpha}(S), k_{E} \in \mathscr{B}_{\alpha}(S)$, and since $\mathscr{B}_{\alpha}(S)$ is complete in the sup norm (Hausdorff [9, p. 269]), we have $B\left(S, A_{\alpha}(S)\right) \subset \mathscr{B}_{\alpha}(S)$. Conversely, each $f \in \mathscr{B}_{\alpha}(S)$ is uniformly approximated by a sequence of step functions in $B\left(S, A_{\alpha}(S)\right.$ ) (Hahn [7, Theorem 34.4.31], Hausdorff [9, p. 280], Kuratowski [10, p. 388]). 
This finishes the proof.

REMARK 1.2. It follows easily from the proof of Theorem 1.1 that (a) $Z_{\alpha}(S)$ is precisely the family of zero sets $f^{-1}(0)$ for $f \in \mathscr{B}_{\alpha}(S)$, and (b) $\mathscr{B}_{\omega_{1}}(S)$ is precisely the family of bounded functions $f: S \rightarrow \boldsymbol{R}$ which are measurable with respect to the $\sigma$-field $Z_{\omega_{1}}(S)$ of Baire sets in $S$.

CoROLlary 1.3. If $S$ is a normal space and $\Sigma$ is the field of all subsets of $S$ which are both $F_{\sigma}$ and $G_{\delta}$ in $S$, then $\mathscr{B}_{1}(S)=B(S, \Sigma)$.

Proof. Obviously, $A_{1}(S) \subset \Sigma$, so it suffices to show that $B(S, \Sigma) \subset$ $\mathscr{B}_{1}(S)$. It is enough only to show $k_{E} \in \mathscr{B}_{1}(S)$ if $E \in \Sigma$. If $E=\bigcup F_{n}=$ $\cap G_{n}$ with $F_{1} \subset F_{2} \subset \cdots$ closed and $G_{1} \supset G_{2} \supset \cdots$ open, choose by Urysohn's lemma continuous $f_{n}$ such that $f_{n}\left(F_{n}\right)=1, f_{n}\left(G_{n}^{c}\right)=0$. Then $\lim f_{n}=k_{E} \in \mathscr{B}_{1}(S)$. This proves the corollary.

If $\Sigma$ is a field of subsets of $S$, denote by $S_{\Sigma}$ the space of all multiplicative linear functionals on $B(S, \Sigma)$, endowed with the $w^{*}$ topology. Then $S_{\Sigma}$ is a compact Hausdorff space, and if for $f \in B(S, \Sigma)$ we define $\hat{f} \in C\left(S_{\Sigma}\right)$ by $\hat{f}(x)=x(f)\left(x \in S_{\Sigma}\right)$, then the map $f \rightarrow \hat{f}$ defines an algebraic isometry of $B(S, \Sigma)$ onto $C\left(S_{\Sigma}\right)$. For each $s \in S$, let $\tau(s) \in S_{\Sigma}$ be the multiplicative linear functional on $B(S, \Sigma)$ obtained by evaluation at $s$. The space $S_{\Sigma}$ is totally disconnected, and the map $E \rightarrow \overline{\tau(E)}(E \in \Sigma)$ defines a Boolean algebra isomorphism of $\Sigma$ onto the field of clopen (closed and open) sets in $S$. Thus $S_{\Sigma}$ is a Stone representation space for $\Sigma$. (See Dunford-Schwartz [5, pp. 311-312] for details regarding this discussion.) We therefore infer from Theorem 1.1 the following representation of $\mathscr{B}_{\alpha}(S)$ (for $S=[0,1]$, see Bade [2, p. 3]):

THEOREM 1.4. $\mathscr{B}_{\alpha}(S)$ is algebraically isometric to a space $C\left(\Omega_{\alpha}\right)$, where $\Omega_{\alpha}$ is a compact totally disconnected Hausdorff space. Furthermore, there exists a function $\tau_{\alpha}: S \rightarrow \Omega_{\alpha}$ such that

(a) $\hat{f}\left(\tau_{\alpha}(s)\right)=f(s)$ for all $s \in S$ (where $\hat{f}$ is the correspondent in $C\left(\Omega_{\alpha}\right)$ to $\left.f \in \mathscr{B}_{\alpha}(S)\right)$, and

(b) $E \rightarrow \overline{\tau_{\alpha}(E)}, E \in A_{\alpha}(S)$, defines a Boolean isomorphism of $A_{\alpha}(S)$ onto the field of clopen sets in $\Omega_{\alpha}$.

2. Baire complemented $C(S)$ spaces.

Definition 2.1. For the Banach space $X$, denote by $X_{1}$ the first Baire space for $X$, i.e., the set of all limits in $X^{* *}$ of $w^{*}$ convergent sequences in $X$ (regarded as a subspace of $X^{* *}$ ). The space $X$ is called Baire complemented if there is a continuous linear projection from $X_{1}$ onto $X$. 


\section{REMARKS 2.2.}

(1) $X_{1}$ is a closed subspace of $X^{* *}$ (McWilliams [12]).

(2) $X$ is Baire complemented if and only if $X$ has a complementary closed subspace in $X_{1}$.

(3) If $X$ and $Y$ are isomorphic Banach spaces and $X$ is Baire complemented, then $Y$ is Baire complemented.

(4) If $X$ is Baire complemented and $Y$ is a complemented subspace of $X$, then $Y$ is Baire complemented. (For if $P: X_{1} \rightarrow X$ and $Q: X \rightarrow Y$ are projections and $J: Y \rightarrow X$ is the inclusion map, then $Q P J^{* *} \mid Y_{1}$ is a projection of $Y_{1}$ onto $Y$.)

(5) If $X=C(S)$ for the compact Hausdorff space $S$, then $X_{1}$ is isometrically identified with $\mathscr{B}_{1}(S)$ by the map $u: \mathscr{B}_{1}(S) \rightarrow X_{1}$ defined by $u(f)(\mu)=\int_{S} f d \mu$ for $f \in \mathscr{B}_{1}(S)$ and $\mu \in X^{*}=C(S)^{*}=M(S)$. (By the Lebesgue dominated convergence theorem, a bounded sequence $f_{n} \in C(S)$ is pointwise convergent on $S$ to an $f \in \mathscr{B}_{1}(S)$ if and only if $f_{n}$ converges $w^{*}$ in $C(S)^{* *}$ to $u(f) \in X_{1}=C(S)_{1}$.) Therefore, $C(S)$ is Baire complemented if and only if $C(S)$ is complemented in $\mathscr{B}_{1}(S)$.

(6) If $T: X \rightarrow Y$ is an isomorphism of $X$ onto $Y$, then $T^{* *} \mid X_{1}$ is an isomorphism of $X_{1}$ onto $Y_{1}$. Thus, by Remark (5), if $S$ and $S^{\prime}$ are compact Hausdorff spaces such that $C(S)$ and $C\left(S^{\prime}\right)$ are isomorphic, then $\mathscr{B}_{1}(S)$ and $\mathscr{B}_{1}\left(S^{\prime}\right)$ are also isomorphic. By a simple induction, $\mathscr{B}_{\alpha}(S)$ and $\mathscr{B}_{\alpha}\left(S^{\prime}\right)$ are isomorphic for all $\alpha$.

The next objective is to establish a topological condition on $S$ which prevents $C(S)$ from being Baire complemented. First, some terminology is needed.

Suppose $S$ and $T$ are compact Hausdorff spaces and $\varphi: S \rightarrow T$ is a continuous map of $S$ onto $T$. An averaging operator for $\varphi$ is a continuous linear map $P: C(S) \rightarrow C(T)$ such that $P(f \circ \phi)=f, f \in C(T)$. If $\left\{t_{\alpha}\right\}$ is a net in $T$ and $t_{\alpha} \rightarrow t$, let

$$
\lim \sup \varphi^{-1}\left(t_{\alpha}\right)=\left\{\begin{array}{l}
s \in S: \text { for each } \alpha_{0} \text { and neighborhood } U \\
\text { of } s, \varphi^{-1}\left(t_{\alpha}\right) \cap U \neq \phi \text { for some } \alpha \geqq \alpha_{0}
\end{array}\right\} .
$$

The set $\lim \sup \varphi^{-1}\left(t_{\alpha}\right)$ is a nonempty compact subset of $\varphi^{-1}(t)$. For the integer $n>1$, define

$$
M_{\varphi}^{(1)}(n)=\left\{\begin{array}{l}
t \in T: \varphi^{-1}(t) \text { contains } n \text { disjoint sets of } \\
\text { the form } \lim \sup \varphi^{-1}\left(t_{\alpha}\right) \text { for nets } t_{\alpha} \rightarrow t
\end{array}\right\} .
$$

For integers $k, n_{1}, \cdots, n_{k}>1$ define, inductively on $k$,

$$
M_{\varphi}^{(k)}\left(n_{1}, \cdots, n_{k}\right)=\left\{\begin{array}{l}
t \in T: \varphi^{-1}(t) \text { contains } n_{k} \text { disjoint sets of } \\
\text { the form } \lim \sup \varphi^{-1} t\left({ }_{\alpha}\right) \text { for nets } t_{\alpha} \rightarrow t \\
\text { such that } t_{\alpha} \in M_{\varphi}^{(k-1)}\left(n_{1}, \cdots, n_{k-1}\right)
\end{array}\right\}
$$


TheOREM 2.3. (S. Z. Ditor [4, p. 204]). If $M_{\varphi}^{(k)}\left(n_{1}, \cdots, n_{k}\right) \neq \phi$ and $P$ is an averaging operator for $\varphi$, then $\|P\| \geqq 1+2 \sum_{i=1}^{k}\left(1-1 / n_{i}\right)$.

Throughout the remainder of this section, $S$ is a compact Hausdorff space and $\Sigma$ is a field of subsets of $S$ such that $C(S) \subset B(S, \Sigma)$. These fields are characterized in the next lemma. A neighborhood base in $S$ is a family $\mathscr{F}$ of subsets of $S$ (not necessarily open) such that if $U$ is open in $S$ and $s \in U$, then for some $E \in \mathscr{F}, s \in \operatorname{int}(E)$ and $E \subset U$.

Lemma 2.4. These are equivalent:

(1) $C(S) \subset B(S, \Sigma)$;

(2) $\Sigma$ contains a neighborhood base;

(3) Any two disjoint closed subsets of S are contained in disjoint members of $\Sigma$.

Proof. (3) $\Rightarrow(1)$ : For $f \in C(S)$ and $\varepsilon>0$, choose $a_{0}<a_{1}<\cdots<a_{n}$ so that $f(S) \subset\left[a_{0}, a_{n}\right]$ and $a_{i}-a_{i-1}<\varepsilon, 1 \leqq i \leqq n$. By (3), pick $A_{0}$, $\cdots, A_{n} \in \Sigma$ with $A_{0}=\phi, A_{n}=S$, and for $1 \leqq i<n,\left\{t: f(t) \leqq a_{i}\right\} \subset A_{i}$ and $\left\{t: f(t) \geqq a_{i+1}\right\} \cap A_{i}=\phi$. If $g=\sum_{i=1}^{n} a_{i} k_{A_{i}-A_{i-1}}$, then $g \in B(S, \Sigma)$ and $\|g-f\|<\varepsilon$, proving (1). (1) $\Rightarrow(3)$ : For $F_{0}, F_{1}$ closed and disjoint pick $f \in C(S)$ with $f\left(F_{0}\right)=0, f\left(F_{1}\right)=1$. Choose by (1) a step function $g \in B(S, \Sigma)$ with $\|g-f\|<1 / 2$. If $A=\{t: g(t)<1 / 2\}$, then $A \in \Sigma$, $F_{0} \subset A$, and $F_{1} \subset A^{\circ} \in \Sigma$, proving (3). (2) $\Leftrightarrow$ (3) by normality and compactness. This proves the lemma.

If $E \subset S$, then $E^{-}=\bar{E}=$ the closure of $E$.

Definition 2.5. For the integer $n>1$, let $\Gamma_{\Sigma}^{(1)}(n)$ be the set of all $x$ in $S$ such that there exist $n$ disjoint sets $G_{1}, \cdots, G_{n} \in \Sigma$ with

$$
x \in \bigcap_{i=1}^{n}\left[\operatorname{int}\left(G_{i}\right)\right]^{-} \text {. }
$$

For the integers $k, n_{1}, \cdots, n_{k}>1$, define, inductively on $k, \Gamma_{2}^{(k)}\left(n_{1}\right.$, $\cdots, n_{k}$ ) be the set of all $x \in S$ such that there exist $n_{k}$ disjoint sets $G_{1}, \cdots, G_{n_{k}} \in \Sigma$ with

$$
x \in \bigcap_{i=1}^{n_{k}}\left[\operatorname{int}\left(G_{i}\right) \cap \Gamma_{\Sigma}^{(k-1)}\left(n_{1}, \cdots, n_{k-1}\right)\right]^{-} .
$$

THEOREM 2.6. If $P$ is a projection of $B(S, \Sigma)$ onto $C(S)$ and $\Gamma_{\Sigma}^{(k)}\left(n_{1}, \cdots, n_{k}\right) \neq \phi$, then $\|P\| \geqq 1+2 \sum_{i=1}^{k}\left(1-1 / n_{i}\right)$.

Proof. Use the notation $\left(\tau: S \rightarrow S_{\Sigma} ; f \rightarrow \hat{f}\right)$ preceding Theorem 1.4. Define the continuous map $\phi$ from $S_{\Sigma}$ onto $S$ by $f(\varphi(x))=\hat{f}(x)$ for $x \in S_{\Sigma}$ and $f \in C(S)$. If $G \subset S$ is open and $x \in \varphi^{-1}(G)$, then there exists a net $x_{\alpha}=\tau\left(s_{\alpha}\right) \rightarrow x$ (since $\left.\overline{\tau(S)}=S_{\Sigma}\right)$, and $\varphi\left(\tau\left(s_{\alpha}\right)\right)=s_{\alpha} \rightarrow \varphi(x) \in G$, 
so that $s_{\alpha}$ is eventually in $G$. Thus $\tau\left(s_{\alpha}\right)$ is eventually in $\tau(G)$, so $x \in \overline{\tau(G)}$. Thus

$$
\phi^{-1}(G) \subset \overline{\tau(G)} \text { for open } G \subset S .
$$

Suppose now that $P: B(S, \Sigma) \rightarrow C(S)$ is a projection. If $Q: C\left(S_{\Sigma}\right) \rightarrow$ $C(S)$ is defined by $Q(\hat{f})=P(f), f \in B(S, \Sigma)$, then $Q$ is an averaging operator for $\varphi$ and $\|Q\|=\|P\|$. The theorem is proved by showing, inductively on $k$, that $\Gamma_{\sim}^{(k)}\left(n_{1}, \cdots, n_{k}\right) \subset M_{\varphi}^{(k)}\left(n_{1}, \cdots, n_{k}\right)$ and applying Ditor's theorem.

For $k=1$, pick $s \in \Gamma^{(1)}(n)$ and disjoint sets $G_{1}, \cdots, G_{n} \in \Sigma$ such that $s \in \bigcap_{i=1}^{n}\left[\operatorname{int}\left(G_{i}\right)\right]^{-}$. In each set int $\left(G_{\imath}\right)$ there is a net $s_{\alpha} \rightarrow s$, and by $(*), \varphi^{-1}\left(s_{\alpha}\right) \subset \varphi^{-1}\left(\operatorname{int}\left(G_{i}\right)\right) \subset \overline{\tau\left(G_{i}\right)}$. Thus $\lim \sup \varphi^{-1}\left(s_{\alpha}\right) \subset$ $\varphi^{-1}(s) \cap \overline{\tau\left(G_{i}\right)}$. But since $E \rightarrow \overline{\tau(E)}$ is a Boolean isomorphism, the sets $\overline{\tau\left(G_{1}\right)}, \cdots, \overline{\tau\left(G_{n}\right)}$ are disjoint. Thus $\varphi^{-1}(s)$ contains $n$ disjoint sets of the form $\lim \sup \varphi^{-1}\left(s_{\alpha}\right)$ for nets $s_{\alpha} \rightarrow s$, i.e., $s \in M_{\psi}^{(1)}(n)$.

Now suppose $k>1$ and $\Gamma_{2}^{(k-1)}\left(n_{1}, \cdots, n_{k-1}\right) \subset M_{\varphi}^{(k-1)}\left(n_{1}, \cdots, n_{k-1}\right)$. Pick $s \in \Gamma_{\Sigma}^{(k)}\left(n_{1}, \cdots, n_{k}\right)$ and disjoint sets $G_{1}, \cdots, G_{n_{k}} \in \Sigma$ such that $s \in \bigcap_{i=1}^{n}\left[\operatorname{int}\left(G_{i}\right) \cap \Gamma_{-}^{(k-1)}\left(n_{1}, \cdots, n_{k-1}\right)\right]^{-}$. In each set int $\left(G_{i}\right)$ there is a net $s_{\alpha} \rightarrow s$ with $s_{\alpha} \in \Gamma_{i}^{(k-1)}\left(n_{1}, \cdots, n_{k-1}\right) \subset M_{\varphi}^{(k-1)}\left(n_{1}, \cdots, n_{k-1}\right)$. As before, $\lim \sup \varphi^{-1}\left(s_{\alpha}\right) \subset \varphi^{-1}(s) \cap \overline{\tau\left(G_{i}\right)}$, and the $\overline{\tau\left(G_{i}\right)}\left(1 \leqq i \leqq n_{k}\right)$ are disjoint. Thus $\varphi^{-1}(s)$ contains $n_{k}$ disjoint lim sup sets of nets of fibers $\varphi^{-1}\left(s_{\alpha}\right)$ for $s_{\alpha} \in M^{(k-1)}\left(n_{1}, \cdots, n_{k-1}\right)$. This means $s \in M^{(k)}\left(n_{1}, \cdots, n_{k}\right)$. This proves the theorem.

REMARK 2.7. A different proof of Theorem 2.6 was given by Amir [1] under the assumption that $\Sigma$ contains an open base for $S$ and is stable under the closure operation. However, Theorem 2.6 is applied below in Corollary 2.10 to fields $\Sigma$ which are not necessarily stable under closure. The preceding proof supplies details for the method outlined by Ditor [4, p. 205].

COROLLARY 2.8. If there exists a nonempty subset $Q \subset S$ such that for every $s \in Q$, there exist disjoint sets $G_{1}, G_{2} \in \Sigma$ with

$$
s \in\left[\operatorname{int}\left(G_{1}\right) \cap Q\right]^{-} \cap\left[\operatorname{int}\left(G_{2}\right) \cap Q\right]^{-},
$$

then $C(S)$ is not complemented in $B(S, \Sigma)$.

Proof. By induction on $k, Q \subset \Gamma_{\Sigma}^{(k)}(2, \cdots, 2)$ for $k=1,2,3, \cdots$. By Theorem 2.6, a projection must then have norm $\geqq 1+k$ for all $k$, an impossibility.

Recall that a compact Hausdorff space is called an $F$-space (GillmanJerison [6]) if disjoint open $F_{\sigma}$ sets always have disjoint closures. This motivates the following terminology. 
Definition 2.9. A compact Hausdorff space $S$ is called strongly non-F if there exists a nonempty subset $Q \subset S$ such that for each $s \in Q$, there exist disjoint open $F_{\sigma}$ sets $G_{1}$ and $G_{2}$ with

$$
s \in \overline{G_{1} \cap Q} \cap \overline{G_{2} \cap Q} .
$$

COROLLARY 2.10. Suppose $\Sigma$ is the field generated by the open $F_{\sigma}$ subsets of the compact space $S$. If $S$ is strongly non-F, then $C(S)$ is not complemented in $B(S, \Sigma)$. In particular, $C(S)$ is not Baire complemented.

Proof. The first statement follows from Corollary 2.8 and Definition 2.9. By Corollary 1.3, $B(S, \Sigma) \subset \mathscr{B}_{1}(S)$, so $C(S)$ is not Baire complemented by Remark 2.2(5).

REMARK. An easy application of Phillips' lemma shows that if $S$ has a nontrivial convergent sequence, then $C(S)$ is not Baire complemented (due to Wells [18]).

There is a partial converse to the preceding corollary. Recall that a compact Hausdorff is called $\sigma$-Stonian, or basically disconnected, if the closure of each open $F_{\sigma}$ set is open. Thus each $\sigma$-Stonian space is an $F$-space. The next theorem gives an analogue for $\sigma$ Stonian spaces of the well-known fact that a compact space $S$ is Stonian (extremally disconnected) if and only if $C(S)$ is the range of a norm 1 projection from $C(S)^{* *}$ (see, e.g., Semadeni $[17, \S \S 25.5$ and 27.1.2]).

THEOREM 2.11. For a compact Hausdorff space S, these are equivalent:

(1) $S$ is $\sigma$-Stonian;

(2) $C(S)$ is Baire complemented by a projection of norm 1 from $\mathscr{B}_{1}(S)$

(3) $C(S)$ is the range of a norm 1 projection from $B(S, \Sigma)$, where $\Sigma$ is the field generated by the open $F_{\sigma}$-sets in $S$;

(4) $C(S)$ is the range of a multiplicative norm 1 projection from $\mathscr{B}_{\omega_{1}}(S)$, the space of all bounded Baire functions on $S$;

(5) $C(S)$ is a $\sigma$-complete lattice.

Proof. The proof goes $(1) \Rightarrow(4) \Rightarrow(2) \Longrightarrow(3) \Rightarrow(5) \Rightarrow(1) . \quad(1) \Longrightarrow(4)$ : Let $S$ be $\sigma$-Stonian. Denote by $\mathscr{B}$ the $\sigma$-field of Baire sets and by $\mathscr{C}$ the field of clopen sets. For each $E \in \mathscr{B}$, there exists a unique $\rho(E) \in \mathscr{C}$ such that $E \Delta \rho(E)$ is meager, and the map $\rho: \mathscr{B} \rightarrow \mathscr{C}$ is a Boolean retraction (Halmos [8, p. 102]). For $s \in S$, let $\delta_{s}$ be the unit point mass measure at $s$. Define $\mu_{s}(E)=\delta_{s}(\rho(E)), E \in \mathscr{B}$, and 
$P: \mathscr{B}_{\omega_{1}}(S) \rightarrow C(S)$ by

$$
(P f)(s)=\int f d \mu_{s}, \quad f \in \mathscr{B}_{\omega_{1}}(S), s \in S .
$$

Observing that $\mathscr{B}_{\omega_{1}}(S)=B(S, \mathscr{B})($ Theorem 1.1) and $C(S)=B(S, \mathscr{C})$, it is easy to check that $P$ is the desired projection.

$(4) \Rightarrow(2) \Rightarrow(3): \quad$ Obvious since $C(S) \subset B(S, \Sigma) \subset \mathscr{B}_{1}(S)$ (Lemma 2.4 and Corollary 1.3).

$(3) \Longrightarrow(5)$ : Let $P: B(S, \Sigma) \rightarrow C(S)$ be a projection of norm 1 , and let $f_{1} \leqq f_{2} \leqq \cdots$ be an increasing bounded sequence in $C(S)$ with $f(s)=\lim f_{n}(s)$. Then $f \in B(S, \Sigma)$ (Hausdorff [9, pp. 279-280 and Theorem 5, p. 270]) and since $P$ is order-preserving (Pelczynski [14, Proposition 1.2]), we obtain $f_{n}=P f_{n} \leqq P f$. If $g \in C(S)$ and $f_{n} \leqq g$, $n=1,2, \cdots$, then $f \leqq g$ and $P f \leqq P g=g$. Therefore $P f=\mathrm{V} f_{n}$, and $C(S)$ is a $\sigma$-complete lattice.

$(5) \Leftrightarrow(1)$ is well-known (Semadeni [17, Proposition 24.7.4]). This concludes the proof.

REMARK. Because of (4), the projections in (2) and (3) can be taken multiplicative.

3. Baire classes over $[0,1]$. In this section the previous results are applied to the Baire classes $\mathscr{B}_{\alpha}([0,1])$, denoted simply by $\mathscr{B}_{\alpha}$. The arguments which follow hold equally well if $[0,1]$ is replaced by any uncountable complete separable metric space. Regarding the representation $\mathscr{B}_{\alpha}=C\left(\Omega_{\alpha}\right)$, the objective is to show that, for $0<$ $\alpha<\omega_{1}, \Omega_{\alpha}$ is strongly non- $F$ (Theorem 3.3). The crucial information is provided by the following result of Lusin in descriptive set theory.

Lemma 3.1 (Lusin). Denote by $\mathscr{F}$ the space of irrational numbers. For each countable ordinal $\alpha$, there exist in the space $\mathscr{I} \times \mathscr{J}$ two disjoint sets of additive class $\alpha$ which are not contained in disjoint sets of ambiguous class $\alpha$.

Proof. Lusin [11, p. 204] or Hahn [7, p. 274].

Lemma 3.2. For every countable ordinal $\alpha$, there exist in the Cantor set $K$ two disjoint subsets $A$ and $B$ of additive class $\alpha$ in $K$ such that, if $A^{\prime} \subset A$ and $B^{\prime} \subset B$ are any subsets with $A-A^{\prime}$ and $B-B^{\prime}$ countable, then $A^{\prime}$ and $B^{\prime}$ are not contained in disjoint sets of ambiguous class $\alpha$.

Proof. We treat the cases $\alpha=1$ and $\alpha>1$ separately. For $\alpha=1$, first notice that every open set $V \subset K$ contains a perfect 
nowhere dense subset (see Kuratowski [10, p. 447]). Let $V_{1}, V_{2}, \cdots$ denote a base of open sets for $K$. Choose perfect nowhere dense sets $A_{1} \subset V_{1}$ and $B_{1} \subset V_{1}-A_{1}$, and, inductively on $n$, let $A_{n}$ and $B_{n}$ be disjoint perfect nowhere dense subsets of $V_{n}-\bigcup_{k=1}^{n-1}\left(A_{k} \cup B_{k}\right)$, $n=2,3, \cdots$. The sets $A=\bigcup_{n=1}^{\infty} A_{n}$ and $B=\bigcup_{n=1}^{\infty} B_{n}$ are disjoint $F_{\sigma}$ sets in $K$ (i.e., additive class 1 ), and if $A^{\prime} \subset A$ and $B^{\prime} \subset B$ with $A-A^{\prime}$ and $B-B^{\prime}$ countable then $A^{\prime}$ and $B^{\prime}$ are still dense in $K$. Thus $A^{\prime}$ and $B^{\prime}$ are not contained in disjoint sets of ambiguous class 1 because, by the Baire category theorem, two dense $G_{\delta}$ sets must intersect.

For $\alpha>1$, first let $D \subset K$ be a denumerable dense set, so that $K-D$ is homeomorphic to $\mathscr{F}$ (Kuratowski [10, p. 441]) and therefore to $\mathscr{F} \times \mathscr{F}$. By the Lusin lemma, there exist in $K-D$ two disjoint sets $A, B$ of additive class $\alpha$ (relative to $K-D$ ) which are not contained in disjoint ambiguous class $\alpha$ sets. Since $K-D$ is a $G_{\delta}$ and $\alpha \geqq 2, A$ and $B$ are additive class $\alpha$ relative to $K$ (see Hahn [7, Proposition 33.4.73]) and are not contained in disjoint ambiguous class $\alpha$ sets in $K$. Since every countable set is ambiguous class 2, no sets $A^{\prime} \subset A$ and $B^{\prime} \subset B$, such that $A-A^{\prime}$ and $B-B^{\prime}$ are countable, can be contained in disjoint ambiguous class $\alpha$ sets. This proves the lemma.

THEOREM 3.3. For each countable ordinal $\alpha>0$, the maximal ideal space $\Omega_{\alpha}$ of the $\alpha$ th Baire class $\mathscr{B}_{\alpha}$ is strongly non-F.

Proof. A set $Q \subset \Omega_{\alpha}$ is exhibited which satisfies Definition 2.9 . Let $I=[0,1]$. Fix the countable ordinal $\alpha \geqq 1$, and define $\tau_{\alpha}: I \rightarrow \Omega_{\alpha}$ as in Theorem 1.4. Now define

$Q=\left\{x \in \Omega_{\alpha}:\right.$ there exist two disjoint open $F_{\sigma}$ sets in $\Omega_{\alpha}, G_{1}$ and $G_{2}$, such that if $W$ is a neighborhood of $x$, then the sets $\tau_{\alpha}^{-1}\left(G_{1} \cap W\right)$ and $\tau_{\alpha}^{-1}\left(G_{2} \cap W\right)$ are uncountable $\}$.

It is first shown that if $K \subset I$ is any homeomorphic image of the Cantor set, then $\overline{\tau_{\alpha}(K)}$ contains a point of $Q$-showing in particular that $Q \neq \phi$. For such $K$, choose two disjoint additive class $\alpha$ sets $A, B$ as guaranteed by Lemma 3.2. Let $\mathscr{F}$ denote the family of compact subsets of $\Omega_{\alpha}$ defined by

$$
\begin{aligned}
\mathscr{F}= & \left\{\overline{\tau_{\alpha}(M)}: \text { either } M \subset A \text { and } A-M\right. \text { is countable } \\
& \text { or } M \subset B \text { and } B-M \text { is countable }\}
\end{aligned}
$$

We show that $\mathscr{F}$ has the finite intersection property. If $M_{1}, \cdots$, $M_{n} \subset A$ and $M_{n+1}, \cdots, M_{p} \subset B$ with $\overline{\tau_{\alpha}\left(M_{i}\right)} \in \mathscr{F}(1 \leqq i \leqq p)$, let $A^{\prime}=$ $\bigcap_{i=1}^{n} M_{i} \subset A$ and $B^{\prime}=\bigcap_{i=n+1}^{p} M_{i} \subset B$. Then $A-A^{\prime}$ and $B-B^{\prime}$ are 
countable, so that $A^{\prime}$ and $B^{\prime}$ are not contained in disjoint ambiguous class $\alpha$ sets. But this implies that $\overline{\tau_{\alpha}\left(A^{\prime}\right)} \cap \overline{\tau_{\alpha}\left(B^{\prime}\right)} \neq \phi$ since by Theorem 1.4, $\Omega_{\alpha}$ is totally disconnected and if $\overline{\tau_{\alpha}\left(A^{\prime}\right)} \cap \overline{\tau_{\alpha}\left(B^{\prime}\right)}=\phi$, then for some clopen set $C, \overline{\tau_{\alpha}\left(A^{\prime}\right)} \subset C, C \cap \overline{\tau_{\alpha}\left(B^{\prime}\right)}=\phi$. But since $E \rightarrow \overline{\tau_{\alpha}(E)}$ is a Boolean isomorphism of $A_{\alpha}(I)$ onto the field of clopen sets in $\Omega_{\alpha}$, we have $C=\overline{\tau_{\alpha}(E)}$ for some $E \in A_{\alpha}(I)$. Then $A^{\prime} \subset E$ and $B^{\prime} \subset E^{c}$, a contradiction. Therefore, $\phi \neq \overline{\tau_{\alpha}\left(A^{\prime}\right)} \cap \overline{\tau_{\alpha}\left(B^{\prime}\right)} \subset \bigcap_{i=1}^{p} \overline{\tau_{\alpha}\left(M_{i}\right)}$, and $\mathscr{F}$ has the finite intersection property.

Now choose $x \in \bigcap\{C: C \in \mathscr{F}\}$. Clearly, $x \in \overline{\tau_{\alpha}(\bar{K})}$. We show $x \in Q$. Since $A$ and $B$ are additive class $\alpha$ sets, there exist sets $A_{1}, A_{2}, \cdots$, $B_{1}, B_{2}, \cdots \in A_{\alpha}(I)$ such that $A=\bigcup A_{n}$ and $B=\bigcup B_{n}$. Define the disjoint open $F_{\sigma}$ sets $G_{1}$ and $G_{2}$ in $\Omega_{\alpha}$ by

$$
G_{1}=\bigcup \overline{\tau_{\alpha}\left(A_{n}\right)}, \quad G_{2}=\bigcup \overline{\tau_{\alpha}\left(B_{n}\right)} .
$$

If $W$ is a neighborhood of $x$, let $E_{1}=\tau_{\alpha}^{-1}\left(G_{1} \cap W\right)$. If $E_{1}$ were only countable, then $\overline{\tau_{\alpha}\left(A-E_{1}\right)} \in \mathscr{F}$, so $x \in \overline{\tau_{\alpha}\left(A-E_{1}\right)}$. Thus $\tau_{\alpha}(t) \in W$ for some $t \in A-E_{1}$, which is a contradiction. This shows $\tau_{\alpha}^{-1}\left(G_{1} \cap W\right)$ is uncountable, and likewise $\tau_{\alpha}^{-1}\left(G_{2} \cap W\right)$ is uncountable. This concludes the proof that for every subset $K \subset I$ homeomorphic to the Cantor set, $\overline{\tau_{\alpha}(K)}$ contains a point of $Q$.

Now choose $x \in Q$, and suppose $G_{1}$ and $G_{2}$ are disjoint open $F_{\sigma}$ sets satisfying the definition of $Q$. Let $W$ be a clopen set containing $x$, and fix attention on $G_{1} \cap W$. Write $G_{1}=\bigcup_{n=1}^{\infty} F_{n}$, where each $F_{n}$ is clopen. We have $\tau_{\alpha}^{-1}\left(G_{1} \cap W\right)=\bigcup \tau_{\alpha}^{-1}\left(F_{n} \cap W\right)$, which is uncountable, so there must exist $n_{0}$ such that $\tau_{\alpha}^{-1}\left(F_{n_{0}} \cap W\right)$ is uncountable. But $F_{n_{0}} \cap W$ is clopen hence $F_{n_{0}} \cap W=\overline{\tau_{\alpha}(E)}$ for some $E \in A_{\alpha}(I)$. Then $E=\tau_{\alpha}^{-1}\left(F_{n_{0}} \cap W\right)$, and the Baire set $E$, being uncountable, must contain a copy $K$ of the Cantor set (Kuratowski [10, p. 447]), and $\overline{\tau_{\alpha}(K)} \subset$ $F_{n_{0}} \cap W$. By what was shown above, $\overline{\tau_{\alpha}(K)}$ contains a point of $Q$, so that $G_{1} \cap W$ contains a point of $Q$. Since $W$ was arbitrarily chosen, $x \in \overline{G_{1} \cap Q}$. Similarly, $x \in \overline{G_{2} \cap Q}$. Therefore, $Q$ satisfies the condition of Definition 2.9, and $\Omega_{\alpha}$ is strongly non- $F$. This concludes the proof of Theorem 3.3.

REMARK 3.4. The preceding proof is more delicate than necessary for $\alpha \geqq 2$. In case $\alpha \geqq 2$, one could simply take $Q=\bigcup\left(\bar{G}_{1} \cap \bar{G}_{2}\right)$, where the union runs over all pairs $\left(G_{1}, G_{2}\right)$ of disjoint open $F_{o}$ sets in $\Omega_{\alpha}$. The "uncountability condition" is automatically satisfied. However, for $\alpha=1$, the proof given seems to be required, and the case $\alpha \geqq 2$ goes along for a free ride. However, we use the nonseparation result of Lusin (Lemma 3.1), which becomes decidedly nontrivial in the case $\alpha \geqq 2$.

Corollary 3.5. For $\alpha<\omega_{1}, \mathscr{B}_{\alpha}$ is not Baire complemented. 
Proof. Corollary 2.10 and Theorem 3.3.

THEOREM 3.6. Suppose $S$ is a compact Hausdorff space which contains an uncountable compact metrizable subset. Then for each countable ordinal $\alpha>0, \mathscr{B}_{\alpha}(S)$ is not Baire complemented.

Proof. Let $K \subset S$ be an uncountable compact metric space. By Milutin's theorem (Pelczynski [14, Theorem 8.5]), $C(K)$ and $C([0,1])$ are isomorphic, so by Remarks $2.2(6), \mathscr{B}_{\alpha}(K)$ and $\mathscr{B}_{\alpha}$ are isomorphic for all $\alpha$. But $\mathscr{B}_{\alpha}$ is not Baire complemented (Corollary 3.5), so $\mathscr{B}_{\alpha}(K)$ is not Baire complemented. (This conclusion can also be proved in the same way as Corollary 3.5, thus avoiding Milutin.)

By the Borsuk-Dugundji theorem (see Pelczynski [14, Theorem 6.6]), there exists an "extension operator" $E: C(K) \rightarrow C(S)$ such that $E$ is an isometry and $E(f) \mid K=f$. Regarding $\mathscr{B}_{\alpha}(K)$ and $\mathscr{B}_{\alpha}(S)$ as subspaces of $C(K)^{* *}$ and $C(S)^{* *}$ under the canonical embedding, we obtain by an easy induction argument that for each $h \in \mathscr{B}_{\alpha}(K)$, $E^{* *}(h) \in \mathscr{B}_{\alpha}(S)$ and $E^{* *}(h) \mid K=h$. That is, $E^{* *}$ extends $E$ to an "extension operator" from $\mathscr{B}_{\alpha}(K)$ into $\mathscr{B}_{\alpha}(S)$. For $f \in \mathscr{B}_{\alpha}(S)$, we have that $f \mid K \subset \mathscr{B}_{\alpha}(K)$, and by defining $P(f)=E^{* *}(f \mid K)$ we obtain a projection $P$ of $\mathscr{B}_{\alpha}(S)$ onto $E^{* *}\left(\mathscr{B}_{\alpha}(K)\right)$, which is isometric to $\mathscr{B}_{\alpha}(K)$. Therefore, $\mathscr{B}_{\alpha}(S)$ can not be Baire complemented since $\mathscr{B}_{\alpha}(K)$ is not Baire complemented (see Remarks 2.2(3) and 2.2(4)). This proves the theorem.

Corollary 3.7. Suppose $S$ is a compact Hausdorff space which contains an uncountable compact metrizable subset. Then for each countable ordinal $\alpha>0, \mathscr{B}_{\alpha}(S)$ is not isomorphic to any complemented subspace of a space $C(\Omega)$ for $\sigma$-Stonian $\Omega$. In particular, $\mathscr{B}_{\alpha}(S)$ is not isomorphic to any complemented subspace of $\mathscr{B}_{\omega_{1}}(S)$.

Proof. The first statement follows from Theorem 2.11 and Remarks 2.2(3) and 2.2(4). For the second statement, represent $\mathscr{B}_{\omega_{1}}(S)$ by Theorem 1.4 as $C(\Omega)$, where $\Omega$ is a totally disconnected compact space whose Boolean algebra of clopen sets is $\sigma$-complete (being isomorphic to the $\sigma$-field of all Baire sets in $S$ ). It is wellknown that such a space $\Omega$ must be $\sigma$-Stonian (see Halmos [8, p. 99]).

REMARK. A Banach space $X$ is called a Grothendieck space if every continuous linear map $T: X \rightarrow c_{0}$ is weakly compact. It is known (see Seever [16, p. 272]) that if $\Omega$ is $\sigma$-Stonian (or even an $F$-space), then every quotient of $C(\Omega)$ is a Grothendieck space. A proof that $\mathscr{B}_{\alpha}\left(\alpha<\omega_{1}\right)$ is not a Grothendieck space, together with the proof of Theorem 3.6, would provide a stronger result than Corollary 3.7. 
However, it is at present unknown whether $\mathscr{B}_{\alpha}$ is a Grothendieck space. In fact, it is even unknown whether the maximal ideal space $\Omega_{\alpha}$ contains any nontrivial convergent sequence (the existence of such a sequence would prevent $\mathscr{B}_{\alpha}=C\left(\Omega_{\alpha}\right)$ from being a Grothendieck space).

Proposition 3.8. Suppose $S$ is a topological space and $\alpha>0$ is an ordinal such that $\mathscr{B}_{\alpha}(S)$ is not Baire complemented. Then $\mathscr{B}_{\alpha}(S)$ is not complemented in $\mathscr{B}_{\beta}(S)$ for any $\beta>\alpha$.

Proof. Use the notation $\left(\tau_{\alpha}: S \rightarrow \Omega_{\alpha} ; f \mapsto \hat{f} ; \mathscr{B}_{\alpha}(S) \approx C\left(\Omega_{\alpha}\right)\right)$ of Corollary 1.4. Define $T: \mathscr{B}_{1}\left(\Omega_{\alpha}\right) \rightarrow \mathscr{B}_{\alpha+1}(S)$ by $T h=h \circ \tau_{\alpha}, h \in \mathscr{B}_{1}\left(\Omega_{\alpha}\right)$. (To check that $T h \in \mathscr{B}_{\alpha+1}(S)$, pick $h_{n} \in C\left(\Omega_{\alpha}\right)$ such that $\lim h_{n}=h \in$ $\mathscr{B}_{1}\left(\Omega_{\alpha}\right)$, and choose $f_{n} \in \mathscr{B}_{\alpha}(S)$ so that $\hat{f}_{n}=h_{n}$. Then $h_{n} \circ \tau_{\alpha}=\hat{f}_{n} \circ \tau_{\alpha}=f_{n}$, $T h(s)=\lim h_{n}\left(\tau_{\alpha}(s)\right)=\lim f_{n}(s)$, and $T h \in \mathscr{B}_{\alpha+1}(S)$.) $\quad$ Suppose there were a projection $P$ from $\mathscr{B}_{\alpha+1}(S)$ onto $\mathscr{B}_{\alpha}(S)$. Define $R: \mathscr{B}_{1}\left(\Omega_{\alpha}\right) \rightarrow C\left(\Omega_{\alpha}\right)$ by $R(h)=(P T(h))^{\wedge}, h \in \mathscr{B}_{1}\left(\Omega_{\alpha}\right)$. Then $R$ is obviously a projection onto $C\left(\Omega_{\alpha}\right)$, contradicting the assumption. Thus no such $P$ can exist. Obviously, therefore, there can be no projection from $\mathscr{B}_{\beta}(S)$ onto $\mathscr{B}_{\alpha}(S)$ for any $\beta>\alpha$. This proves the proposition.

We obtain as a corollary the following result of W. G. Bade [2]:

CoRollary 3.9. For the countable ordinals $\alpha<\beta, \mathscr{B}_{\alpha}([0,1])$ is not complemented in $\mathscr{B}_{\beta}([0,1])$.

Proof. Corollary 3.5 and Proposition 3.8.

Remark 3.10. By Theorem 3.6 and Proposition 3.8, the above corollary remains true if $[0,1]$ is replaced by any compact Hausdorff space which contains an uncountable compact metrizable subset.

Corollary 3.7 implies that $\mathscr{B}_{\alpha}$ is not isomorphic to $\mathscr{B}_{\omega_{1}}$ for $\alpha<\omega_{1}$. It follows from [3, Corollary 8, p. 336] that $\mathscr{B}_{1}$ is not isomorphic to $\mathscr{B}_{\alpha}$ for $\alpha>1$. Although it is unknown whether $\mathscr{B}_{\alpha}$ and $\mathscr{B}_{\beta}$ are isomorphic for $1<\alpha<\beta<\omega_{1}$, it is shown in the next theorem that they are not isometric.

THEOREM 3.11. If $0<\alpha<\beta \leqq \omega_{1}$, then $\mathscr{B}_{\alpha}$ and $\mathscr{B}_{\beta}$ are not isometric.

Proof. By Theorem 1.4, $\mathscr{B}_{\alpha}=C\left(\Omega_{\alpha}\right)$ and $\mathscr{B}_{\beta}=C\left(\Omega_{\beta}\right)$, where $\Omega_{\alpha}$ (resp. $\Omega_{\beta}$ ) is a totally disconnected compact Hausdorff space whose field of clopen sets is Boolean isomorphic to the field $A_{\alpha}$ of ambiguous class $\alpha$ sets (resp. to the field $A_{\beta}$ of ambiguous class $\beta$ sets). If 
$\mathscr{B}_{\alpha}$ and $\mathscr{B}_{\beta}$ are isometric, then $\Omega_{\alpha}$ and $\Omega_{\beta}$ are homeomorphic by the Banach-Stone theorem, and so $A_{\alpha}$ and $A_{\beta}$ are isomorphic Boolean algebras. Let $\rho: A_{\alpha} \rightarrow A_{\beta}$ be a Boolean isomorphism. Since the algebras $A_{\alpha}$ and $A_{\beta}$ are purely atomic, $\rho$ must be induced by a point map, i.e., there must exist a permutation $\pi$ of $[0,1]$ such that $\rho(E)=\pi(E), E \in A_{\alpha}$. If $G \subset[0,1]$ is open, then $G \in A_{\beta}$ so $\pi^{-1}(G) \in A_{\alpha}$ and $\pi^{-1}(G)$ is a Borel set. Thus $\pi$ is Borel measurable, so there must exist a first category $F_{\sigma}$ set $F$ so that $\pi \mid F^{\circ}$ is continuous (Kuratowski [10, p. 400]). Since $F^{c}$ is an uncountable $G_{\delta}, F^{c}$ contains a copy $K$ of the Cantor set. Thus $\pi \mid K$ is continuous and one-toone, therefore a homeomorphism. Thus $\pi(E) \in A_{\alpha}$ if $E \in A_{\alpha}(K)$ since a homeomorphism preserves class. But this is a contradiction, because $\pi(K)$, being: homeomorphic to $K$, contains subsets in $A_{\beta}-A_{\alpha}$ (Hausdorff [9, p. 207]), each of which is assumed to be of the form $\pi(E)$ for $E \in A_{\alpha}(K)$. This proves the theorem.

REMARK. In connection with the above, it follows from a result of Rosenthal [15, p. 242, Remark 3] that the dual spaces $\mathscr{B}_{\alpha}^{*}\left(1 \leqq \alpha \leqq \omega_{1}\right)$ are all linearly isometric to each other and to $\left(l^{\infty}\right)^{*}$.

THEOREM 3.12. If $S$ and $T$ are uncountable complete separable metric spaces, then $\mathscr{B}_{\omega_{1}}(S)$ and $\mathscr{\mathscr { S }}_{\omega_{1}}(T)$ are isometric by an algebraic isometry which maps $\mathscr{B}_{\alpha}(S)$ onto $\mathscr{B}_{\alpha}(T)$ for all infinite ordinals $\alpha$.

Proof. There exists a "Borel isomorphism" $\varphi$ between $S$ and $T$ which is class 1 in both directions (Kuratowski [10, p. 451]), i.e., a bijection $\varphi: S \rightarrow T$ so that $\varphi$ and $\varphi^{-1}$ are both class 1 . Since $\varphi$ and $\varphi^{-1}$ change the class of a set by at most $1, \varphi$ and $\varphi^{-1}$ in fact preserve class at the first limit ordinal, and therefore at every higher level. That is, $E \in A_{\alpha}(S)$ if and only if $\varphi(E) \in A_{\alpha}(T), \alpha \geqq \omega_{0}$. Thus $A_{\alpha}(S)$ and $A_{\alpha}(T)$ are isomorphic Boolean algebras, so that $B\left(S, A_{\alpha}(S)\right) \approx$ $B\left(T, A_{\alpha}(T)\right.$ ), i.e., $\mathscr{B}_{\alpha}(S) \approx \mathscr{B}_{\alpha}(T)$ for $\alpha \geqq \omega_{0}$ by Theorem 1.1. This concludes the proof.

\section{REFERENCES}

1. D. Amir, Projections onto continuous function spaces, Proc. Amer. Math. Soc., 15 (1964), 396-402.

2. W. G. Bade, Complementation problems for the Baire classes, Pacific J. Math., 45 (1973), 1-11.

3. F. K. Dashiell and J. Lindenstrauss, Some examples concerning strictly convex norms on $C(K)$ spaces, Israel J. Math., 16 (1973), 329-342.

4. S. Z. Ditor, Averaging operators in $C(S)$ and lower semicontinuous sections of continuous maps, 'Trans. Amer. Math. Soc., 175 (1973), 195-208.

5. N. Dunford and J. T. Schwartz, Linear Operators, Part I, Interscience, New York, 1966. 
6. L. Gillman and M. Jerison, Rings of Continuous Functions, Van Nostrand Reinhold, New York, 1960.

7. Hans Hahn, Reelle funktionen, Leipzig, 1932 (repr. Chelsea, New York, 1948).

8. P. R. Halmos, Lectures on Boolean Algebras, Van Nostrand Mathematical Studies No. 1, D. Van Nostrand, Princeton, 1963.

9. Felix Hausdorff, Set Theory, 2d ed., Chelsea, New York, 1962.

10. K. Kuratowski, Topology, Vol. I, Academic Press, New York, 1966.

11. N. Lusin, Lecons sur les ensembles analytiques, Paris, 1930 (repr. Chelsea, New York, 1972).

12. R. D. McWilliams, A note on weak sequential convergence, Pacific J. Math., 12 (1962), 333-335.

13. I. P. Natanson, Theory of functions of a real variable, Vol. II, Ungar, New York, 1960.

14. A. Pelczynski, Linear extensions, linear averagings, and their applications to linear topological classification of spaces of continuous functions, Dissertationes Math. Rozprawy Mat., 58 (1968).

15. H. P. Rosenthal, On injective Banach spaces and the spaces $L^{\infty}(\mu)$ for finite measures $\mu$, Acta Math., 124 (1970), 205-248.

16. G. L. Seever, Measures on F-spaces, Trans. Amer. Math. Soc., 133 (1968), 267-280.

17. Z. Semadeni, Banach spaces of continuous functions, Vol. I, Monografie Matematyczne, Tom 55, PWN, Warszawa, 1971.

18. B. B. Wells, Jr., Uncomplemented spaces of continuous functions and weak compactness of measures, Ph.D. Thesis, University of California, Berkeley, 1967.

Received August 15, 1973. This is a part of the author's Ph.D. thesis prepared at the University of California, Berkeley, under the direction of William G. Bade, and supported in part by NSF Grant GP-29012.

University of CALIFornia, Los ANgeles 
